ARTICLE

https://doi.org/10.1038/s41467-019-13353-5

\title{
Sugar-mediated regulation of a c-di-GMP phosphodiesterase in Vibrio cholerae
}

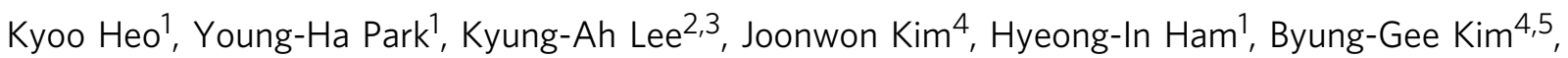
Won-Jae Lee ${ }^{2,3} \&$ Yeong-Jae Seok (D) ${ }^{1 \star}$

Biofilm formation protects bacteria from stresses including antibiotics and host immune responses. Carbon sources can modulate biofilm formation and host colonization in Vibrio cholerae, but the underlying mechanisms remain unclear. Here, we show that ElIAGlc, a component of the phosphoenolpyruvate (PEP):carbohydrate phosphotransferase system (PTS), regulates the intracellular concentration of the cyclic dinucleotide c-di-GMP, and thus biofilm formation. The availability of preferred sugars such as glucose affects EllA Glc phosphorylation state, which in turn modulates the interaction of EllAGlc with a c-di-GMP phosphodiesterase (hereafter referred to as PdeS). In a Drosophila model of $V$. cholerae infection, sugars in the host diet regulate gut colonization in a manner dependent on the PdeS-ElIA Glc interaction. Our results shed light into the mechanisms by which some nutrients regulate biofilm formation and host colonization.

\footnotetext{
${ }^{1}$ School of Biological Sciences and Institute of Microbiology, Seoul National University, Seoul 08826, Republic of Korea. ${ }^{2}$ School of Biological Sciences and National Creative Research Initiative Center for Hologenomics, Seoul National University, Seoul 08826, Republic of Korea. ${ }^{3}$ Institute of Molecular Biology and Genetics, Seoul National University, Seoul 08826, Republic of Korea. ${ }^{4}$ School of Chemical and Biological Engineering and Institute of Molecular Biology and Genetics, Seoul National University, Seoul 08826, Republic of Korea. ${ }^{5}$ Institute of Engineering Research, Seoul National University, Seoul 08826, Republic of Korea. *email: yjseok@snu.ac.kr
} 
$\mathrm{M}$ ost bacterial species can form biofilms, which are multicellular communities embedded in a self-produced polymeric matrix. The ability of pathogenic bacteria to form biofilms facilitates their colonization and persistence in the host due to the evasion of the immune response and increased resistance to many antimicrobials ${ }^{1,2}$. In addition, it has been reported that dispersed cells exhibit acute virulence and efficient transmission to other hosts, indicating that an appropriate transition to planktonic cells from these sessile lifestyles is a crucial factor in their pathogenicity ${ }^{3-6}$. Thus, it is conceivable that sophisticated mechanisms should have evolved for pathogenic bacteria to regulate the transition between these two lifestyles in response to various environmental cues ${ }^{7}$.

It has been shown that glucose and some other sugars in the environment induce the multilayer biofilm formation and the sugars promoting biofilm formation are substrates of the phosphoenolpyruvate (PEP):carbohydrate phosphotransferase system (PTS) in V. cholerae ${ }^{7}$. This multi-component system mediates the transport of various sugars including glucose in $V$. cholerae, and these PTS sugars are concomitantly phosphorylated during transport $^{8,9}$. The PTS consists of two general components, enzyme I (EI) and histidine phosphocarrier protein (HPr) that participate in the transport of most PTS sugars, and several sugarspecific components collectively known as enzyme IIs (EIIs). EI and HPr transfer a phosphoryl group from PEP to EII components, which finally phosphorylate PTS carbohydrates during their translocation across the membrane.

In addition to its role in carbohydrate transport and phosphorylation, the PTS acts as an efficient signal transduction system, which can sense the availability of carbohydrates in the environment and thereby regulates various cellular functions ${ }^{10}$. Phosphorylation of the PTS components usually increases in the absence and decreases in the presence of a PTS carbohydrate such as glucose $\mathrm{e}^{11,12}$. Depending on their phosphorylation state, the PTS components regulate various sugar-related phenotypes by interacting with their cognate partners ${ }^{13}$. Although the effects of the PTS on biofilm formation have been reported in several studies $9,14,15$, the regulation mechanism of biofilm formation by the PTS has not been fully elucidated.

In enteric bacteria such as Escherichia coli, EIIA ${ }^{\text {Glc }}$ (encoded by $\mathrm{crr}$ ) is known to play multiple regulatory roles: dephosphorylated EIIA $^{\text {Glc }}$ inhibits non-PTS sugar permeases, such as lactose permease and stimulates the fermentation/respiration switch protein FrsA ${ }^{13,16}$, whereas only phosphorylated EIIA Glc stimulates adenylate cyclase and thus increases the concentration of cAMP ${ }^{17}$, which is known to suppress biofilm formation in V. cholerae ${ }^{18-20}$. An interesting difference between the E. coli and V. cholerae PTS lies in the substrate specificity of EIIA Glc. While EIIA ${ }^{\text {Glc }}$ is specific for glucose in E. coli, its V. cholerae ortholog is shared among several PTS sugars, such as $\mathrm{N}$-acetylglucosamine, trehalose, and sucrose as well as glucose ${ }^{21}$. Therefore, it could be assumed that EIIA $^{\text {Glc }}$ may have distinct regulatory roles in response to several PTS sugars depending on the species. Unlike in E. coli, dephosphorylated EIIA Glc of Vibrio vulnificus inhibits flagella assembly and hence motility, allowing it to efficiently consume a preferable sugar in the environment 22,23 . This regulation of the transition from a motile to non-motile lifestyle mediated by EIIA Glc might also act on biofilm formation processes on the abiotic or biotic surfaces.

The regulatory functions of EIIA ${ }^{\text {Glc }}$ on biofilm formation were suggested in $V$. cholerae in previous studies. EIIA Glc was shown to interact with $\mathrm{MshH}$, a homolog of E. coli $\mathrm{CsrD}^{14}$, and a following study showed that dephosphorylated EIIA Glc activates CsrB/C turnover and increases the amount of $\mathrm{CsrA}^{24}$, which is known to be a negative regulator of biofilm formation in several bacterial species $^{25,26}$. It was also reported that cAMP, the reaction product of adenylate cyclase which is regulated by EIIA ${ }^{\mathrm{Glc}}$, and its receptor protein (CRP) directly and indirectly represses the expression of the diguanylate cyclase $\mathrm{CdgA}$, which positively regulates biofilm formation in the $V$. cholerae C1552 strain ${ }^{20,27}$. While the exogenous addition of cAMP represses the biofilm formation also in the $V$. cholerae MO10 strain, EIIA Glc was shown to activate biofilm formation in the presence of exogenous cAMP in this strain ${ }^{28}$. Collectively, these findings suggest the additional regulatory role of EIIA Glc on biofilm formation.

Here, we explored the molecular mechanism of how the biofilm formation is affected by carbon sources in $V$. cholerae. We find that EIIA Glc interacts with and modulates the activity of a $c$ di-GMP phosphodiesterase (PDE) depending on its phosphorylation state and thereby regulates biofilm formation in the aquatic and host environment. We propose that EIIA Glc functions as a PTS sugar-responsive regulator of the c-di-GMPsignaling pathway and determines whether to disperse from or stay in the biofilm in response to carbohydrates.

\section{Results}

EIIA $^{\text {Glc }}$ interacts with an EAL domain-containing protein in $V$. cholerae. In many bacteria, EIIA ${ }^{\text {Glc }}$ has been implicated in various sugar-dependent regulatory functions ${ }^{13}$. While it was suggested that EIIA ${ }^{\text {Glc }}$ participates in the regulation of biofilm formation in the presence of PTS carbohydrates in V. cholerae ${ }^{9,15}$, no operative mechanisms were yet offered. To examine the effects of EIIA ${ }^{\text {Glc }}$ on biofilm formation, we measured biofilm production in the presence and absence of glucose. While wild-type V. cholerae had a higher level of biofilm formation in the presence of glucose than its absence, which was consistent with previous reports ${ }^{29,30}$, a crr-deficient mutant exhibited no difference between the two conditions (Fig. 1a). Interestingly, while the $\mathrm{crr}$ mutant had a similar growth (Supplementary Fig. 1), this mutant exhibited a higher level of biofilm formation compared to the wild-type strain in LB medium (Fig. 1a), which is contrary to a previous study 9 . The exogenous addition of cAMP did not significantly alter the sugar effect, indicating that the regulation of biofilm formation by EIIA Glc is independent of cAMP. These results led us to search for the regulator of biofilm formation, which transduces the sugar signal by directly interacting with EIIA ${ }^{\text {Glc }}$ in $V$. cholerae.

To find a new interaction partner of EIIA ${ }^{\text {Glc }}$, we performed ligand fishing experiments using hexahistidine-tagged EIIA Glc (His-EIIA $^{\text {Glc }}$ ) as bait ${ }^{22}$. Total proteins extracted from wild-type $V$. cholerae O1 biovar El Tor N16961 cells grown overnight at $37^{\circ} \mathrm{C}$ were mixed with TALON metal-affinity resin in the absence and presence of purified His-EIIA Glc. His-EIIA Glc was dephosphorylated by adding glucose or phosphorylated by adding PEP to the mixtures. After several washes, total proteins bound to the resins were eluted with $200 \mathrm{mM}$ imidazole and analyzed by SDS-PAGE and staining with Coomassie brilliant blue R (Fig. 1b). In repeated experiments, we could find three protein bands migrating with apparent molecular masses of approximately 100,90 , and $60 \mathrm{kDa}$, respectively, that were significantly and reproducibly enriched in the fraction containing both His-EIIA Glc and glucose (lane 2). Peptide mapping of these proteins following in-gel tryptic digestion revealed that the protein band migrating at $\sim 100 \mathrm{kDa}$ corresponded to VC2072, an ortholog of the insulindegrading enzyme IDE (VcIDE) ${ }^{31}$, and the band at $\sim 60 \mathrm{kDa}$ to VCA1085, an ortholog of the flagella assembly protein FapA $(\mathrm{VcFapA})^{22}$. Since IDE and FapA were already reported to interact with EIIA ${ }^{\text {Glc }}$ in $V$. vulnificus ${ }^{22,31}$, the elution of the two proteins only in the fraction containing His-EIIA Glc indicates the reliability of this ligand fishing method (compare lanes 1 and 2). Interestingly, the band at $\sim 90 \mathrm{kDa}$ was identified as a putative 
a

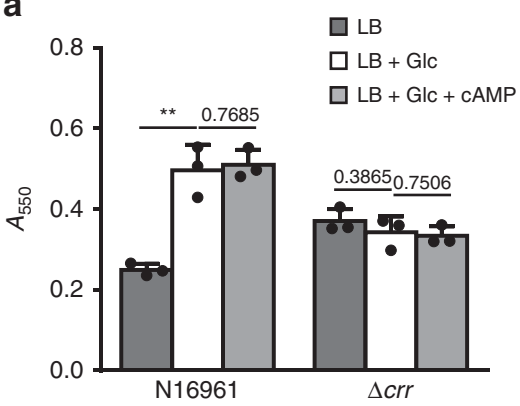

b

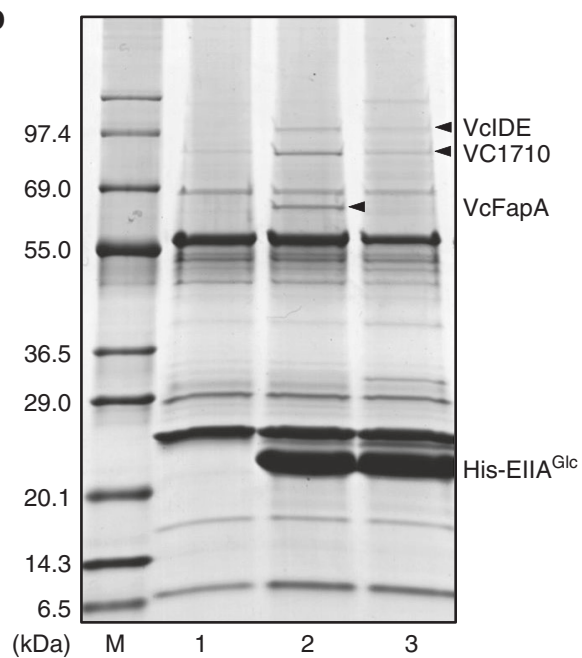

C

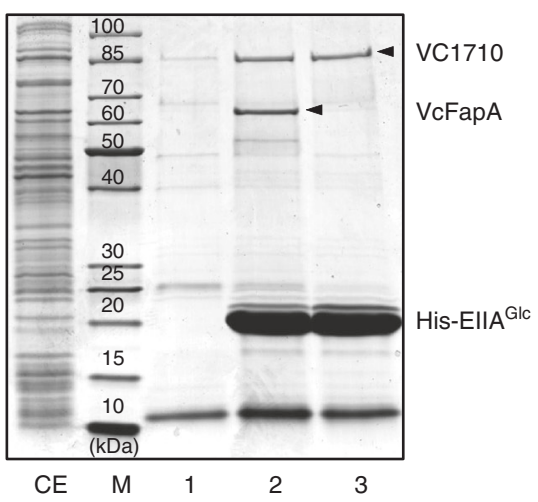

d

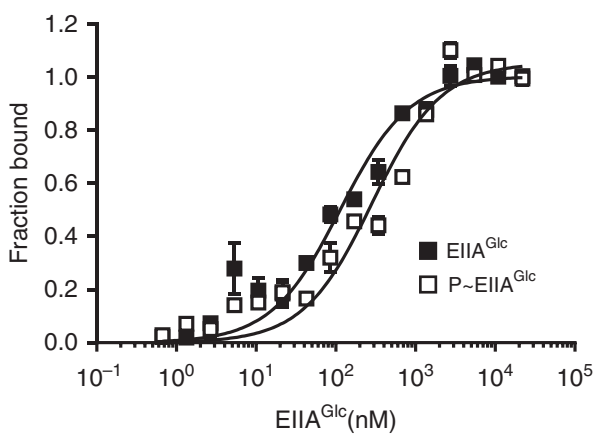

Fig. 1 Specific interaction of VC1710 with ElIA Glc a The biofilm-forming activity of wild-type V. cholerae N16961 and an otherwise isogenic $\Delta$ crr mutant was measured in LB alone or supplemented with glucose or glucose and CAMP, as indicated. Biofilm formation was assessed following the static growth of $V$. cholerae cells for $23 \mathrm{~h}$ using a crystal violet staining method ${ }^{61,62}$. The stained biofilm formation was determined at $550 \mathrm{~nm}$. Statistical significance was assessed using Student's $t$-test ( $p$-values $>0.05$ were presented, ${ }^{\star \star} p$-value $<0.01$ ). Shown are the means and SD ( $n=3$, independent measurements). b Ligand fishing experiment was carried out to find proteins interacting with His-EllAGlc. Crude extract prepared from $V$. cholerae O1 biovar El Tor N16961 cells was mixed with buffer A (lane 1) or $100 \mu \mathrm{g}$ of purified His-EIIA Glc (lanes 2 and 3). The extract containing His-EIIA Glc was supplemented with either $2 \mathrm{mM}$ glucose to dephosphorylate ElIAGlc (lane 2) or $2 \mathrm{mM}$ PEP to phosphorylate ElIAGlc (lane 3). Each mixture was subjected to TALON metal affinity chromatography and proteins bound to the column were analyzed as described in the "Methods" section. c A mixture of $E$. coli cell lysates expressing recombinant VC1710 and VCA1085 (V. cholerae FapA) (lane CE) was mixed with buffer A or His-EllA Glc and subjected to TALON metal affinity chromatography as in panel b. d The binding affinities of VC1710 with either dephosphorylated (black square) or phosphorylated ElIA Glc (white square) were measured using NanoTemper Monolith NT.115pico. The dissociation constants $\left(K_{d}\right)$ of VC1710 complexed with dephosphorylated and phosphorylated EIIA ${ }^{G l c}(P \sim E I I A G l c)$ were obtained from three technical replicates. Shown are the means and SD $(n=3$, independent measurements).

EAL domain-containing c-di-GMP PDE encoded by $v c 1710$. The EAL domain is responsible for degrading bis- $\left(3^{\prime}-5^{\prime}\right)$-cyclic diguanosine monophosphate (c-di-GMP) to $\mathrm{pGpG}^{32}$, which is known to repress biofilm formation and induce biofilm dispersion. Thus, we assumed that the interaction of EIIA ${ }^{\text {Glc }}$ with this EAL domain protein might be implicated in biofilm formation as a response to the sugar signal.

Interactions of a PTS component with its target proteins are usually dependent on the phosphorylation state of that PTS component. While the specific interaction between IDE and EIIA $^{\text {Glc }}$ was reported to be independent of the phosphorylation

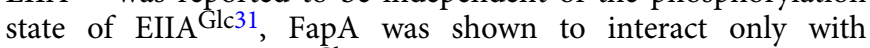
dephosphorylated EIIA Glc in $V$. vulnificus ${ }^{22}$. In accordance with these previous studies, VcFapA was not co-eluted with HisEIIA Glc in the fraction incubated with PEP (lane 3 in Fig. 1b), while the VcIDE band was clearly detected in the eluted fraction containing phosphorylated His-EIIA ${ }^{\text {Glc }}$. Since VC1710 band was detected in the two fractions incubated with EIIA ${ }^{\text {Glc }}$ and either glucose or PEP, we assumed that VC1710 interacts with both dephosphorylated and phosphorylated EIIAGlc. To validate the ligand-fishing data, His-EIIAGlc, EI, and HPr was mixed with glucose or PEP, and the phosphorylation state of His-EIIA ${ }^{\text {Glc }}$ was confirmed by its mobility shift in an SDS-PAGE gel first ${ }^{33}$. Then a mixture of $E$. coli cell extracts expressing recombinant $\mathrm{VC} 1710$ and VcFapA (lane CE) was added and subjected to protein affinity pull-down assays to determine their interaction with either form of EIIA Glc (lanes 2 and 3 in Fig. 1c). As expected, while VcFapA interacted only with dephosphorylated EIIA Glc, VC1710 appeared to interact with both the dephosphorylated and phosphorylated forms of EIIA ${ }^{\mathrm{Glc}}$.

For quantitative analysis of the binding affinity of VC1710 for EIIA $^{\text {Glc }}$, the dissociation constants $\left(K_{\mathrm{d}}\right)$ of VC1710 complexed with dephosphorylated and phosphorylated EIIA Glc $\left(\mathrm{P} \sim \mathrm{EIIA}^{\mathrm{Glc}}\right.$ ) were measured by microscale thermophoresis (MST) experiments. VC1710 showed a slightly higher affinity toward the dephosphorylated form of EIIA Glc $\left(K_{\mathrm{d}}=114.1 \pm 13.7 \mathrm{nM}\right)$ than toward phosphorylated EIIA ${ }^{\text {Glc }}(286.7 \pm 39.5 \mathrm{nM})$ (Fig. 1d). This tight interaction of VC1710 with EIIA ${ }^{\text {Glc }}$ led us to assume that the physiological form of VC1710 exists as a complex with EIIA $^{\text {Glc }}$ and the activity of VC1710 can be influenced by the phosphorylation state of EIIA Glc, as exemplified by the interaction of adenylate cyclase with EIIA Glc in E. coli ${ }^{17}$. 
EIIA $^{\text {Glc }}$ regulates c-di-GMP PDE activity of VC1710. An analysis of the primary structure of $\mathrm{VC} 1710$ indicated that this protein consists of a PAS-sensing domain in the N-terminus and an EAL domain predicted to encode a c-di-GMP PDE in the C-terminus ${ }^{34}$. It has also been previously reported that VC1710 binds c-di-GMP ${ }^{35}$, suggesting its function in c-di-GMP metabolism. To determine whether VC1710 possesses PDE activity, we purified VC1710 and performed an in vitro PDE activity assay by reverse-phase HPLC. The concentration of remaining c-di-GMP decreased with a concomitant increase in the product $\mathrm{pGpG}$ after incubation with $\mathrm{VC1710}$, and we could therefore confirm that VC1710 exhibited the predicted c-di-GMP hydrolytic activity (Fig. 2a, b). Then, to investigate if the PDE activity of VC1710 was affected by its interaction with EIIA ${ }^{\text {Glc }}$, dephosphorylated or phosphorylated EIIA ${ }^{\text {Glc }}$ was added to the reaction mixture. The phosphorylation states of EIIA Glc could be successfully modulated by incubation with EI, $\mathrm{HPr}$, and PEP, as shown in the SDS-PAGE analysis (Fig. 2c). When phosphorylated EIIA Glc was mixed with VC1710, the PDE activity was higher than that in the absence of EIIA ${ }^{\text {Glc }}$. However, no c-di-GMP was digested in the reaction incubated with dephosphorylated EIIA Glc, suggesting that dephosphorylated EIIA Glc inactivates the PDE activity of VC1710. To elucidate the kinetic properties of the VC1710 protein, we determined $K_{\mathrm{m}}$ and $V_{\max }$ values based on three independent activity assays. When c-di-GMP was reacted with
VC1710 alone, the $K_{\mathrm{m}}$ value was $\sim 4.58 \mu \mathrm{M}$. However, when phosphorylated EIIAGlc was added to the VC1710 reaction mixture, the $K_{\mathrm{m}}$ value for c-di-GMP decreased to $1.30 \mu \mathrm{M}$ (Supplementary Fig. 2). Given that the $K_{\mathrm{d}}$ values for c-di-GMPbinding proteins ranged up to several $\mu \mathrm{M}$ and the $K_{\mathrm{m}}$ values of PDEs ranged from $60 \mathrm{nM}$ to several $\mu \mathrm{M}^{36}$, the $K_{\mathrm{m}}$ values of VC1710 for c-di-GMP appear to be biochemically and physiologically relevant to the control of the c-di-GMP level and thereby related phenotypes in vivo. To exclude the possibility of carrier protein effect, we performed the same experiment without EIIA $^{\text {Glc }}$ or in the presence of the same amount of BSA, and no measurable effect was detected (Supplementary Fig. 3). The analytical western blot data (Supplementary Fig. 4) shows that the number of the EIIA Glc protein in a $V$. cholerae cell is more than 100 times higher than that of the $\mathrm{VC1710}$ protein. We, therefore, conclude that VC1710 is always present in a complex form with EIIA ${ }^{\text {Glc }}$ in the cell and that the activity of VC1710 is entirely dependent on the phosphorylation state of EIIA Glc. As the phosphorylation state of EIIA Glc was altered in response to the type of sugars ${ }^{11}$, we named VC1710 as PdeS (for Sugarresponsive PDE).

From the MST assay, we could measure the dissociation constant of the PdeS/c-di-GMP complex to be $\sim 8.7 \mu \mathrm{M}$ (Fig. $2 \mathrm{~d}$ ), which is comparable with the $K_{\mathrm{m}}$ value of PdeS toward c-di-GMP. Interestingly, however, we could not detect any interaction of c-di-
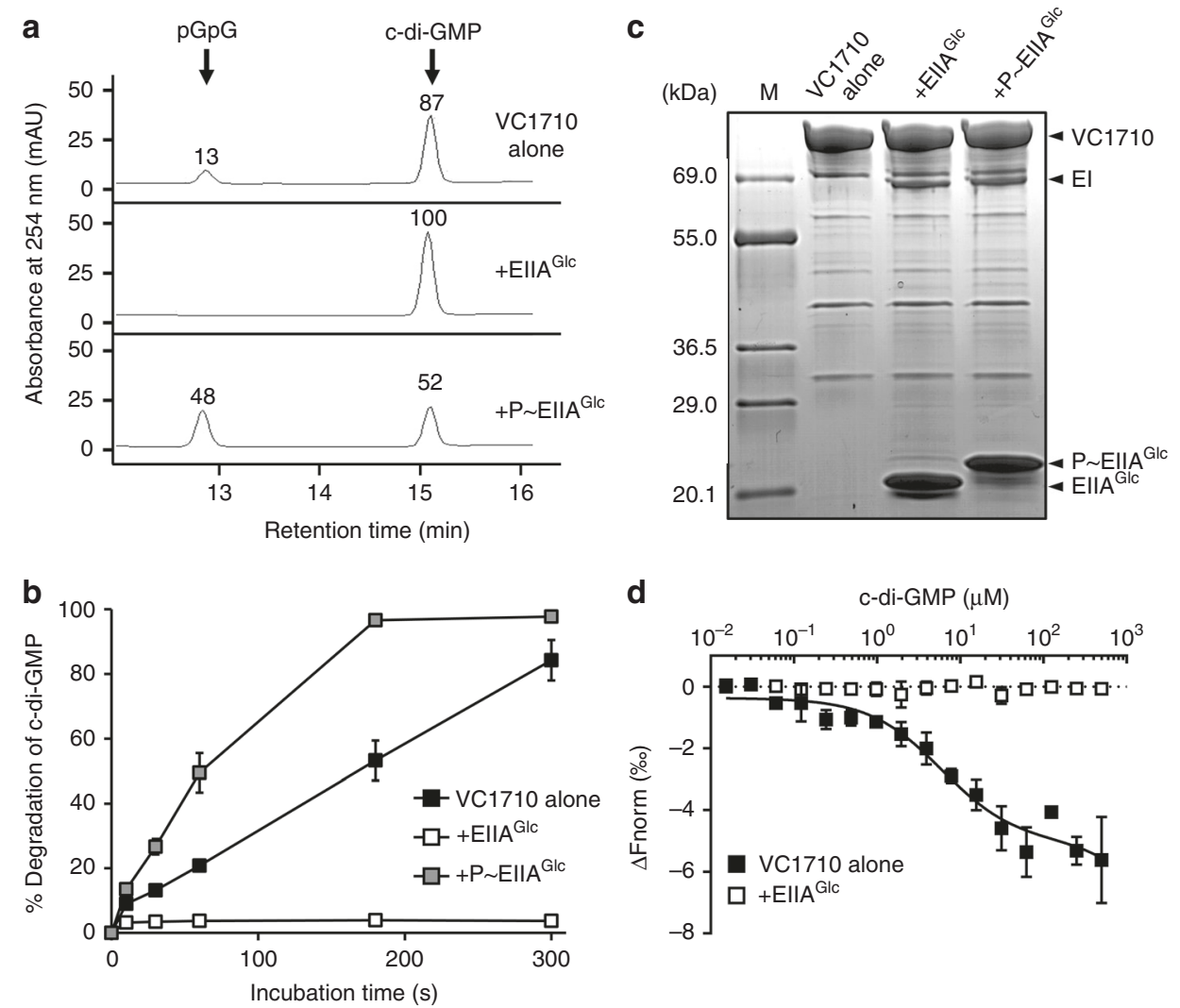

Fig. 2 EllAGlc modulates the PDE activity of VC1710. a The c-di-GMP phosphodiesterase (PDE) activity of VC1710 (4.1 $\mu M)$ was assayed in a reaction mixture containing $20 \mu \mathrm{M}$ of c-di-GMP and $0.4 \mu \mathrm{M}$ of El and HPr in the absence or presence of purified EllA Glc $(17.1 \mu \mathrm{M})$. EllA Glc in the reaction mixture was phosphorylated by adding $2 \mathrm{mM}$ PEP. The reaction mixtures were applied to a Supercosil LC-18-T HPLC column, and the remaining C-di-GMP and produced pGpG were quantified by measuring absorbance at $254 \mathrm{~nm}$. b The c-di-GMP hydrolysis activity of VC1710 was assessed in the absence (black square) and presence of ElIAGlc (white and gray squares) as in panel a and c-di-GMP and pGpG were analyzed at 0, 10, 30, 60, 180, and 300 s. c The phosphorylation state of EIIA Glc in panels $\mathbf{a}$ and $\mathbf{b}$ was confirmed by SDS-PAGE and staining with Coomassie brilliant blue R. $\mathbf{d}$ The binding affinities of PdeS with c-di-GMP in the absence (black square) or presence (white square) of dephosphorylated EllAGlc were measured using NanoTemper Monolith NT.115 ${ }^{\text {pico. }}$. An excess amount $(5 \mu \mathrm{M})$ of ElIA Glc was added to the reaction mixture to ensure all the VC1710 existed as a complex with EllA Glc. Shown are the means and SD ( $n=3$, independent measurements). 
GMP with PdeS in the presence of dephosphorylated EIIA Glc, indicating that PdeS becomes inaccessible to c-di-GMP when it forms a complex with the dephosphorylated form of EIIA Glc (Fig. 2d). Therefore, it could be assumed that the tight binding of dephosphorylated EIIA ${ }^{\text {Glc }}$ inactivates the PDE activity of PdeS by blocking the accessibility of c-di-GMP to the active site.

EIIA $^{\text {Glc }}$ regulates the activity of PdeS in vivo. To test whether PdeS can also digest c-di-GMP in the cell, we compared intracellular concentrations of c-di-GMP between the wild-type and a pdeS-deficient mutant strain $(\Delta p d e S)$ of $V$. cholerae N16961 (Fig. 3a). Because EIIAGlc in cells growing in LB medium is mostly in its phosphorylated form (Fig. 4a), which stimulates the PDE activity of PdeS, the cells grown in the buffered LB medium were used to extract c-di-GMP. Measurement using LC-MS/MS revealed that $\Delta p d e S$ cells had an $\sim 2.5$-fold higher concentration of c-di-GMP than wild-type cells as expected. In addition, the $\Delta p d e S$ strain had a higher expression level of the Vibrio polysaccharide synthesis ( $v p s$ ) operon and thus formed significantly more biofilm than the wild-type strain, which is consistent with previous reports showing that c-di-GMP induces biofilm formation ${ }^{37,38}$ (Fig. 3a, b; Supplementary Fig. 5a). We found that an alanine substitution mutant in the EAL domain of PdeS (PdeS(E450A)) resulted in the complete abolishment of the PDE activity (Supplementary Fig. 3 ). While the $\Delta p d e S$ strain carrying an expression vector for wild-type PdeS exhibited similar levels of biofilm formation and $v p s$ expression with those of the wild-type strain, the mutant strain expressing PdeS(E450A) did not complement these phenotypic changes (Supplementary Figs. 5b and 6). Thus, we concluded that PdeS regulates biofilm formation through its PDE activity.
Then, to confirm whether the regulation of the PdeS activity by EIIA ${ }^{\text {Glc }}$ also operates in $V$. cholerae cells, we constructed a dephosphomimetic mutant (H91A) of $\mathrm{crr}$ encoding EIIA ${ }^{\mathrm{Glc}}$ on the chromosome and performed biofilm formation assays (Supplementary Fig. 7a). While we observed that the dephosphomimetic crr mutant showed increased biofilm formation compared to the wild-type strain, this stimulatory effect was not observed in a pdeS deletion mutant. EIIA Glc is shared for several membrane-bound enzyme IIBCs including those specific for glucose, $N$-acetylglucosamine, sucrose, and trehalose in $V$. cholerae $^{9}$ and phosphorylatable EIIA ${ }^{\text {Glc }}$ is indispensable for a variety of physiological processes including the regulation of global transcription factors such as CRP and Mlc. Therefore, we assume that the phenotype of this chromosomal crr mutant might be due to indirect pleiotropic effects of the mutation. For this reason, we tried to see the effect of EIIAGlc dephosphorylation on biofilm formation by increasing the level of the dephosphomimetic mutant of EIIA Glc, while minimizing the perturbation of the overall PTS activity. Therefore, we compared biofilm formation and the intracellular level of c-di-GMP of the wild-type strain carrying an expression vector for wild-type EIIA $^{\text {Glc }}$ with the wild-type strain carrying an expression vector for EIIA ${ }^{\mathrm{Glc}}(\mathrm{H} 91 \mathrm{~A})$, and observed increased biofilm formation and the c-di-GMP level in the latter strain (Fig. 3c; Supplementary Fig. 7), which is in accordance with the result obtained by the chromosomal mutation of the crr gene. Together our data show that dephosphorylated EIIA ${ }^{\text {Glc }}$ inactivates the PDE activity of PdeS and thereby increases the c-di-GMP level in vivo. Thus, it could be assumed that EIIA ${ }^{\text {Glc }}$ can modulate biofilm formation by controlling the c-di-GMP level through direct interaction with PdeS. a

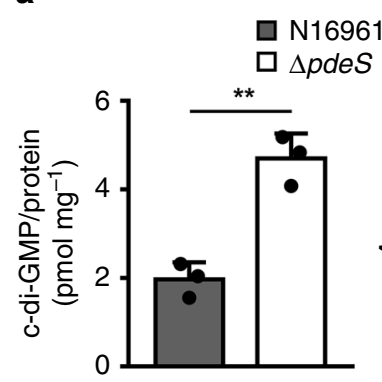

b
N16961

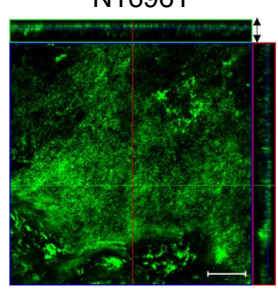

$z=27 \mu \mathrm{m}$
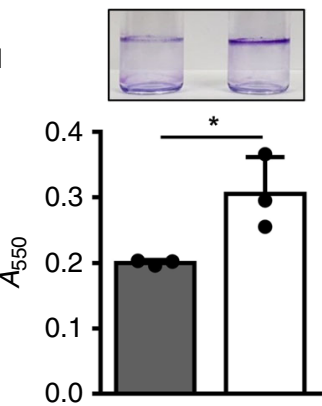

$\Delta p d e S$

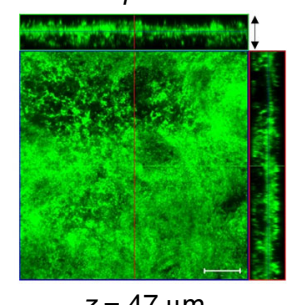

C

$\square$ N16961/pJK-EIIA Glc

$\square$ N16961/pJK-EIIA Glc $(\mathrm{H} 91 \mathrm{~A})$

$\square$ DpdeS/pJK-EIIA Glc

\$ $\Delta$ pdeS/pJK-EIIA ${ }^{\mathrm{Glc}}(\mathrm{H} 91 \mathrm{~A})$

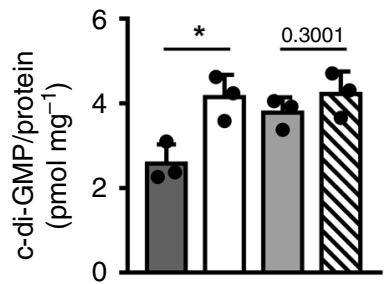

Fig. 3 PdeS regulates the c-di-GMP level and biofilm formation. a The intracellular c-di-GMP concentration and the biofilm-forming activity of wild-type $\mathrm{N} 16961$ and a $\Delta$ pdeS strain were measured in the buffered LB medium. The concentration of c-di-GMP extracted from the $V$. cholerae cells was determined using LC-MS/MS, and normalized with total protein contents. Biofilm formation was assessed following static growth of $V$. cholerae cells for $23 \mathrm{~h}$ using a crystal violet staining method 61,65 . The biofilm formation was determined at $550 \mathrm{~nm}$. b Biofilm formation was visualized using confocal laser scanning microscopy. Scale bar: $50 \mu \mathrm{m}$. c The effect of ElIAGlc on the c-di-GMP hydrolysis activity of PdeS was assessed in vivo. The intracellular c-di-GMP concentration and the biofilm-forming activity of wild-type and $\Delta p d e S V$. cholerae strains harboring a pBAD-based expression vector for either wild-type EIIA Glc or dephosphomimetic mutant ElIA ${ }^{\text {Glc }}(\mathrm{H} 91 \mathrm{~A})$ were determined in the presence of $0.1 \%$ arabinose as described in panel a. Statistical significance was assessed using Student's $t$-test ( $p$-values $>0.05$ were presented, ${ }^{\star} p$-value $<0.05$, ${ }^{\star \star} p$-value $\left.<0.01\right)$. Shown are the means and SD $(n=3$, independent measurements). 
a
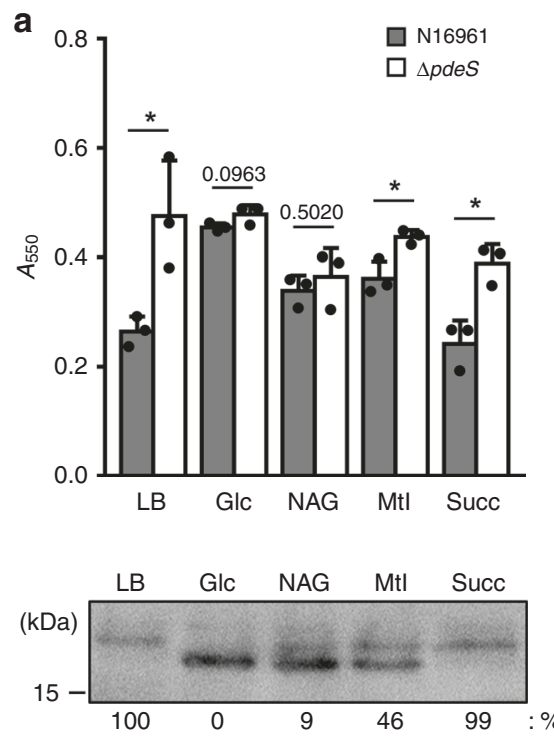

b

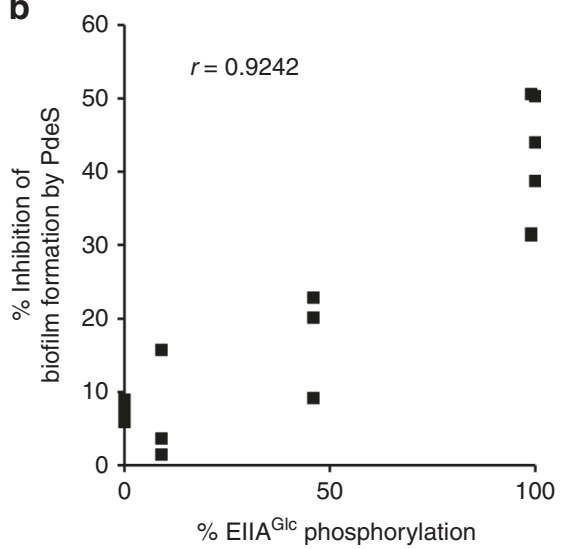

Fig. 4 PdeS regulates biofilm formation depending on carbon sources. a The level of biofilm formation was compared between wild-type N16961 and a $\Delta$ pdeS strain in the presence of various carbon sources in buffered LB medium as in Fig. 3. At the same time, the phosphorylation state of EllAGlc was determined by western blot using anti-EIIA Glc mouse serum after SDS-PAGE. Statistical significance was assessed using Student's $t$-test $\left({ }^{\star} p\right.$-value $<0.05$, p-values > 0.05 were presented). Glc, glucose; NAG, N-acetylglucosamine; Mtl, mannitol; Succ, Succinate. b The inhibitory effect of PdeS on the biofilm formation was plotted as a function of ElIA Glc phosphorylation. Based on the data in panel $\mathbf{a}$, the degree of biofilm formation of wild-type cells was divided by that of the $\Delta$ pdeS mutant cells. The percentage of biofilm formation inhibition by PdeS was then calculated by subtracting the resultant value from 1 and multiplying by 100 , then plotted as a function of the percentage of phosphorylated ElIA Glc over total ElIA Glc. The correlation between the sugar-mediated phosphorylation state of ElIA Glc and biofilm formation was assessed using Pearson's correlation coefficient ( $r=0.9242, p<0.005)$. Shown are the means and SD ( $n=3$, independent measurements).

EIIA $^{\text {Glc }}$ regulates biofilm formation depending on carbon sources. PTS components including EIIA ${ }^{\text {Glc }}$ can have a different phosphorylation state depending on the availability of PTS substrates. As the PDE activity of PdeS and thus the intracellular concentration of c-di-GMP are modulated depending on the phosphorylation state of EIIA ${ }^{\text {Glc }}$, we examined whether biofilm formation can be influenced by carbohydrates encountered by $V$. cholerae in their environmental niches. Thus, we performed a biofilm formation assay in the presence of various carbon sources in $\mathrm{LB}$ medium. To exclude the effect of the $\mathrm{pH}$ decrease due to fermentation of sugar, we buffered LB medium with $40 \mathrm{mM}$ potassium phosphate, $\mathrm{pH}$ 7.0. At the same time, we determined the in vivo phosphorylation state of EIIA ${ }^{\text {Glc }}$ in each medium (Fig. 4a). Consistent with previously reported results in E. coli and V. vulnificus ${ }^{11,22}$, EIIA Glc was mostly dephosphorylated in glucose-containing medium, while mostly phosphorylated in LB medium without additional carbon source. We could also find a significant positive correlation between the degree of EIIA Glc phosphorylation and the inhibitory effect of PdeS on biofilm formation (Fig. 4b). These data imply that the modulation of biofilm formation by PdeS is dependent on the phosphorylation state of EIIA ${ }^{\text {Glc }}$, which is determined by the environmental carbon source.

EIIA Glc regulates biofilm formation and host gut colonization. $V$. cholerae is associated with various hosts, including arthropods, insect eggs, and unicellular eukaryotes as well as humans ${ }^{39}$. Recently, the interaction between $V$. cholerae and the fruit fly Drosophila melanogaster has been intensively studied, since this arthropod model acts as a disease reservoir in nature ${ }^{40}$ and is also simple but has similar physiological features and anatomical structures with mammalian infection models ${ }^{41}$. It has been reported that Vibrio exopolysaccharide (VPS)-dependent biofilm formation is indispensable for attachment and colonization in the
Drosophila intestine in a quorum sensing-defective $V$. cholerae $\operatorname{strain}^{42,43}$. Therefore, we assumed that VPS might also play a critical role in the intestinal colonization in the N16961 strain which carries a natural frame-shift mutation in the hapR gene encoding the quorum-sensing master regulator HapR. As the expression of the $v p s$ operon was controlled by c-di-GMP, which is the substrate of PdeS, we assumed that PdeS might also play a role in the regulation of intestinal colonization. Since mannitol transport by $V$. cholerae requires mannitol-specific EII but not EIIA $^{\text {Glc }}$ in spite of its structural similarity with glucose, the phosphorylation state of EIIA ${ }^{\text {Glc }}$ is different in the presence of the two PTS sugars (Fig. 4a). Therefore, we chose glucose and mannitol as representative sugars leading to dephosphorylation and $\sim 50 \%$ phosphorylation of EIIA Glc, respectively, to evaluate the EIIA ${ }^{\text {Glc }}$ phosphorylation state-dependent regulation of the colonization efficiency of $V$. cholerae. To investigate whether a sugar-dependent change in EIIA ${ }^{\text {Glc }}$ phosphorylation influences bacterial colonization in the intestine through the modulation of PdeS activity, flies were fed 5\% sugar (glucose or mannitol) solution containing $\sim 10^{6}$ cells $_{\mu \mathrm{l}^{-1}}$ of the $V$. cholerae N16961 strain for $24 \mathrm{~h}$, and subsequently fed the same sugar solution without bacteria for $9 \mathrm{~h}$. These flies were then surfacesterilized, homogenized in $1 \mathrm{ml}$ of PBS buffer and spread on an agar plate for the quantification of the colonization of $V$. cholerae in vivo by measuring colony-forming units (CFUs). While the CFU of the $\Delta p d e S$ strain was not affected by the sugar source, the CFU of the wild-type strain was significantly lower in flies fed mannitol compared to flies fed glucose (Fig. 5a). In flies fed mannitol, the wild-type strain gave significantly lower CFU levels compared to the $\Delta p d e S$ strain. Therefore, we could assume that, in the presence of mannitol, phosphorylated EIIA ${ }^{\text {Glc }}$ stimulates the PdeS activity to decrease the c-di-GMP level and thereby biofilm formation.

To determine whether the effect of PdeS on intestinal colonization is mediated by the regulation of biofilm formation, 
a

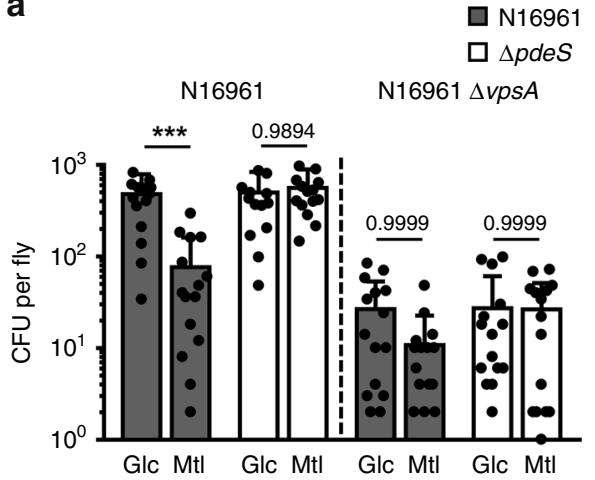

b

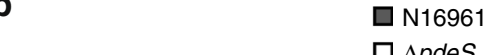

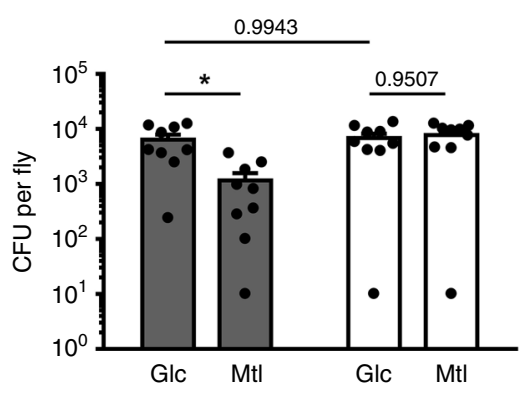
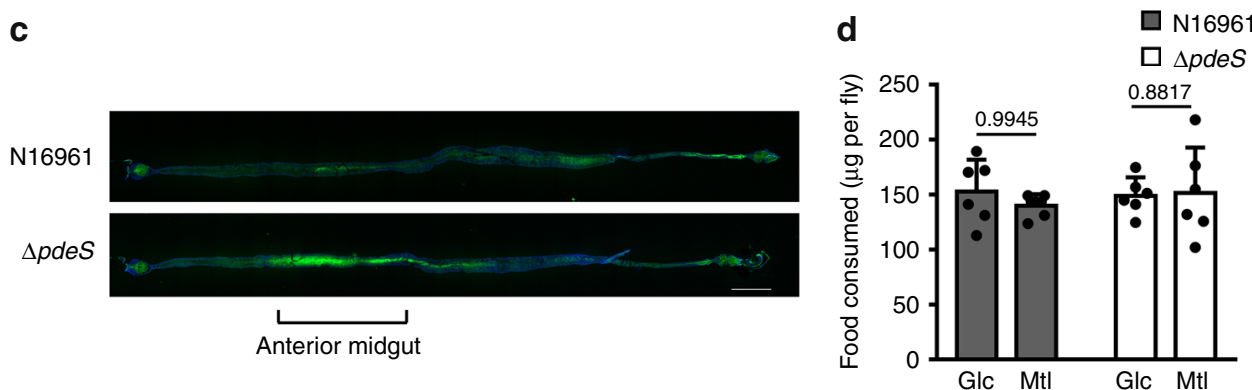

Fig. 5 PdeS regulates intestinal colonization depending on the host diet. a Flies were orally administered a $5 \%$ sugar solution containing the indicated $\mathrm{V}$. cholerae strains for $24 \mathrm{~h}$, and fed a sterile sugar solution for another $9 \mathrm{~h}$. The homogenate of the surface-sterilized fly was spread on a selective medium for $V$. cholerae and colony-forming units (CFU) were measured. $\mathbf{b}$ Intestinal colonization was assessed for a shorter infection period. Flies were orally administered a $5 \%$ sugar solution containing the indicated $V$. cholerae strains for $2 \mathrm{~h}$, and fed a sterile sugar solution for another $2 \mathrm{~h}$. c The $\mathrm{V}$. cholerae colonization on the alimentary canal was visualized using confocal laser scanning microscopy. Flies were exposed to $V$. cholerae strains harboring a GFP expression vector in the presence of $5 \%$ mannitol for $2 \mathrm{~h}$ and, after incubation in a sterile medium for another $2 \mathrm{~h}$, the whole intestines were dissected and visualized. Shown are the representative images from three independent experiments on different days. Scale bar: $500 \mu \mathrm{m}$. $\mathbf{d}$ Food consumption was measured by dye uptake using FD\&C Blue \#1 dye during bacterial infection 64 . Shown are the means and SD $(n=6$, independent measurements). Data was analyzed using a two-way ANOVA followed by Tukey's post hoc test ( ${ }^{\star} p$-value $<0.05,{ }^{\star \star \star} p$-value $<0.005, p$-values $>0.05$ were presented).

we repeated the colonization experiment with $\triangle v p s A$ and $\Delta v p s A$ pdeS mutants (Fig. 5a). As reported previously ${ }^{42,43}$, mutation of $v p s A$ resulted in a significantly reduced $(\sim 5 \%)$ intestinal colonization in flies compared to the wild-type strain. Interestingly, the inhibitory effect of PdeS on intestinal colonization was not seen in this $v p s A$-mutant strain. Thus, it could be assumed that VPS-dependent biofilm formation is important for the regulation of intestinal colonization by PdeS.

Then, to assess whether PdeS affects the earlier stage of $V$. cholerae infection of the Drosophila intestine, we infected flies orally with $5 \%$ sugar solution containing $\sim 10^{8}$ cells $\mu \mathrm{l}^{-1}$ of wildtype $V$. cholerae N16961 or its pdeS mutant for $2 \mathrm{~h}$. After feeding flies with bacteria-free 5\% sugar solution for another $2 \mathrm{~h}$ and surface sterilization, we quantified the intestinal colonization of $V$. cholerae by measuring CFUs (Fig. 5b). While the CFU of the $\Delta p d e S$ strain showed no difference depending on the sugar source, the CFU of the wild-type strain was significantly lower in flies fed mannitol compared to flies fed glucose. Therefore, our data indicates that PdeS may regulate the initial phase of intestinal colonization of $V$. cholerae.

To determine which compartment(s) $V$. cholerae colonizes and to examine the physiological relevance of the host diet-dependent colonization, flies were exposed to the $V$. cholerae N16961 strain carrying a GFP expression vector in the presence of 5\% mannitol, and their colonization on the whole alimentary canal was visualized (Fig. 5c). Although the amounts of ingested bacteria (as estimated by the ingested amounts of bacteria-containing sugar solutions) were similar between wild-type and $\Delta p d e S$ strains (Fig. 5d), more intensive colonization was detected in flies infected with the $\Delta p d e S$ strain than in flies infected with the wild-type strain, especially in the anterior midgut, which is the main site of digestion and nutrient absorption ${ }^{44}$ in our infection condition (Fig. 5c). This result is consistent with the CFU data (Fig. 5b), implying that PdeS modulates the colonization efficiency of $V$. cholerae depending on the host diet, which is consistent with its sugar-dependent regulation of c-di-GMP and biofilm formation.

\section{Discussion}

The pathogen Vibrio cholerae, like other bacteria, has various niches including natural seawater, copepod, chironomid egg masses, and the human host ${ }^{39}$. The ability to sense and respond to each environment is the crucial factor for adaptation and propagation of bacteria. In many bacteria, the PTS is one of the sensory systems that regulate multiple metabolic pathways in response to the availability of carbon or nitrogen nutrients in diverse environments ${ }^{45}$. Previous studies suggested that the PTS in $V$. cholerae also has regulatory functions in biological processes, such as biofilm formation ${ }^{14,28}$, colonization in the mammal host ${ }^{9}$, chitin utilization, and natural competence ${ }^{46}$. Because most of these regulations are mediated by protein-protein interactions ${ }^{13}$, the characterization of the interaction network of the PTS is vital for understanding the orchestrated cellular responses to environmental changes. In this study, we found a new interaction partner of EIIA ${ }^{\text {Glc }}$, the c-di-GMP PDE PdeS which hydrolyzes the ubiquitous bacterial second messenger c-diGMP. As the c-di-GMP-signaling pathway is involved in many behaviors, such as biofilm formation, motility, and toxin production, this interaction is expected to be an important key to reveal many nutrient-related phenotypes. 
Many studies suggested that c-di-GMP induces the transition from motile to sessile mode by binding to and regulating various receptors involved in biofilm formation ${ }^{27,47}$. Biofilm matrix serves as a barrier against various environmental stresses, which enables bacteria to prosper as long as sufficient nutrients are available. However, once nutrients become scarce, bacterial cells detach and disperse from this community by reducing the production of this polymeric matrix. Several studies have reported that nutrient deprivation decreases biofilm formation and induces biofilm dispersion by decreasing intracellular c-di-GMP ${ }^{48-50}$. The interaction between EIIAGlc and PdeS is expected to be one of these regulatory mechanisms.

It is notable that modulation of c-di-GMP hydrolytic activity of PdeS by EIIAGlc is comparable to the regulation of cAMP synthetic activity of adenylate cyclase ${ }^{17}$. In several bacteria, only the phosphorylated form of EIIA Glc is known to stimulate cAMP synthesis ${ }^{13,17,22}$. Similarly, our data in this study show that dephosphorylated EIIAGlc strongly inhibits c-di-GMP hydrolysis, whereas its phosphorylated form stimulates c-di-GMP hydrolysis in $V$. cholerae. The copy number of the EIIAGlc protein was reported to be $20,000-30,000$, while that of adenylate cyclase to be $<10$ in $E$. coli ${ }^{51}$. According to our analytical western blot results, the copy number of EIIA Glc in a $V$. cholerae cell was estimated to be similar to that in E. coli and that of the PdeS protein to be 29-153 (Supplementary Fig. 4). Because the number of EIIA Glc is significantly higher than that of PdeS and adenylate cyclase, EIIA Glc appears to simultaneously control the amount of c-di-GMP and cAMP, yet in the opposite direction, in $V$. cholerae. As the cAMP-CRP complex has been reported to inhibit biofilm formation by differentially regulating the expression of several c-di-GMP-metabolizing enzymes ${ }^{18,20}$, it was expected that the cAMP pathway would mitigate the regulatory effect of PdeS on biofilm formation. However, the expression level of PdeS was not changed by the sugar type (Supplementary Fig. 8). Moreover, the PdeS-mediated regulation of biofilm formation was quantitatively correlated to the phosphorylation state of EIIA Glc (Fig. 4). Therefore, we concluded that although the intracellular level of cAMP and c-di-GMP was controlled at the same time, the sugar-dependent c-di-GMPregulatory pathway is hardly affected by the cAMP-signaling pathway in V. cholerae N16961. In addition, it could be assumed that the indirect interplay between the cAMP and c-di-GMPsignaling pathways might play only a minor role, if any, in the sugar-dependent regulation of biofilm formation in this strain. In previous studies, different effects of cAMP-CRP on biofilm formation have been reported among various $V$. cholerae strains. While biofilm formation was negatively regulated by the cAMP-CRP complex in the C1552 and C6728 strains $^{20,52}$, the supplementation of the growth medium with various concentrations of cAMP had no effect on the total growth and biofilm accumulation by a crr mutant of the MO10 strain ${ }^{28}$. Herein, we report that the sugar-dependent regulation of biofilm formation is not affected by cAMP-CRP in the N16961 strain. This may simply be the result of strain differences, as $V$. cholerae is known to undergo genetic drift in laboratory culture ${ }^{53}$.

During the host infection, the intracellular concentration of c-di-GMP in $V$. cholerae is changed along its infection stage ${ }^{54}$. Also, the c-di-GMP pool fluctuates in response to various external signals, choosing the fittest infection strategy depending on the environmental condition ${ }^{55}$. Thus, the sophisticated regulation of c-di-GMP contents appears to be a prerequisite for a successful propagation and transmission to new hosts throughout the infection cycle. In this respect, our findings provide a new insight into how pathogenic bacteria cope with fluctuating nutritional conditions including those encountered during passage through the intestinal tract of the host.

\section{Methods}

Bacterial strains, plasmids, and culture conditions. The bacterial strains and plasmids used in this study are listed in Supplementary Table 1. Construction of the deletion strains was performed as described previously ${ }^{56}$. V. cholerae strains were cultured in Luria-Bertani medium.

Purification of overexpressed proteins. While EI, HPr, and EIIA Glc were expressed in a ptsHIcrr-deleted E. coli ER2566 strain, other proteins were expressed in wild-type ER2566 by adding $1 \mathrm{mM}$ IPTG. His-tagged proteins were purified using TALON metal-affinity resin (Takara Bio.) according to the manufacturer's instructions. After His-tagged proteins were eluted with $200 \mathrm{mM}$ imidazole, the fractions containing His-tagged proteins were pooled and concentrated using Amicon Ultracel-3K centrifugal filters (Merck Millipore). To increase the purity of proteins and remove imidazole, the concentrated pool was chromatographed on a Hiload 16/60 Superdex $200 \mathrm{pg}$ column (GE Healthcare) equilibrated with buffer A (25 mM HEPES-NaOH (pH 7.6), containing $100 \mathrm{mM} \mathrm{NaCl}, 10 \mathrm{mM} \beta$-mercaptoethanol, and $10 \%$ glycerol).

Ligand fishing using metal-affinity beads. Ligand-fishing experiments were performed as described previously with minor modifications to find a new interaction partner of EIIA Glc23,57. V. cholerae O1 biovar El Tor N16961 cells grown at $37^{\circ} \mathrm{C}$ overnight at LB $(200 \mathrm{ml})$ were harvested and resuspended in buffer A. Cells were then disrupted by three passages through a French pressure cell at 8000 psi. After centrifugation at $10,000 \times g$ for $20 \mathrm{~min}$ at $4{ }^{\circ} \mathrm{C}$, the supernatant was mixed with $100 \mu \mathrm{g}$ of His-EIIA ${ }^{\mathrm{Glc}}$ or buffer A as control in the presence of TALON metalaffinity resin in a $15-\mathrm{ml}$ tube, then incubated at $4^{\circ} \mathrm{C}$ for $30 \mathrm{~min}$. His-EIIA Glc was dephosphorylated by adding glucose or phosphorylated by adding PEP to the mixtures. After brief washes with buffer A containing $10 \mathrm{mM}$ imidazole, the bound proteins were eluted with buffer A containing $200 \mathrm{mM}$ imidazole and analyzed by SDS-PAGE using a 4-20\% gradient Tris-glycine gel (KOMA biotech) and staining with Coomassie brilliant blue R. Protein bands specifically bound to the His-tagged bait protein were excised from the gel, and in-gel digestion and peptide mapping of the tryptic digests were performed using MALDI-TOF $\mathrm{MS}^{57,58}$.

c-di-GMP PDE activity assay using HPLC. c-di-GMP PDE activity was determined by measuring the remaining c-di-GMP and produced $\mathrm{pGpG}$ after the reaction ${ }^{59}$. The reaction contained $20 \mathrm{mM}$ Tris $-\mathrm{HCl}(\mathrm{pH} 8.0), 50 \mathrm{mM} \mathrm{NaCl}, 5 \mathrm{mM}$ $\mathrm{MgCl}_{2}, 0.5 \mathrm{mM}$ EDTA, and $20 \mu \mathrm{M}$ c-di-GMP in addition to PdeS in a total volume of $40 \mu$ l. To test the effect of the phosphorylation state of EIIA ${ }^{\text {Glc }}$ on PDE activity, EIIA Glc and $0.4 \mu \mathrm{M}$ of EI and HPr were added in the presence or absence of PEP. The phosphorylation states of EIIA ${ }^{\text {Glc }}$ were confirmed by SDS-PAGE and staining with Coomassie brilliant blue $\mathrm{R}^{12}$. The enzymatic reaction was started by the addition of c-di-GMP and allowed to proceed at $37^{\circ} \mathrm{C}$. Aliquots of each reaction were taken at appropriate times and reactions were stopped by adding $10 \mathrm{mM}$ $\mathrm{CaCl}_{2}$. These mixtures were boiled for $5 \mathrm{~min}$ and centrifuged. Then $20 \mu \mathrm{l}$ of each supernatant was injected into a Supelcosil LC-18-T column (Sigma-Aldrich) using an Agilent HP1200 HPLC system (Agilent Technology). The separations of pGpG and c-di-GMP were performed by gradient elution at a flow rate of $1.0 \mathrm{ml} \mathrm{min}^{-1}$ and chromatograms were recorded at $254 \mathrm{~nm}$. The gradient program was: $0-5 \mathrm{~min}$, isocratic elution with $100 \mathrm{mM}$ potassium phosphate, $\mathrm{pH} 6.0$ (A); 5-13 min, linear gradient to $70 \% \mathrm{~A}$ and $30 \%$ methanol (B); $13-16 \mathrm{~min}$, isocratic at $\mathrm{B} ; 16-18 \mathrm{~min}$, linear gradient to $\mathrm{A} ; 18-20 \mathrm{~min}$, isocratic at $\mathrm{A}$.

Determination of the intracellular concentration of c-di-GMP. V. cholerae was cultivated in LB medium $(100 \mathrm{ml})$ to $\mathrm{OD}_{600} \sim 1.0$, and centrifuged at $4000 \times g$ for 10 min. The cell pellet was resuspended in $500 \mu \mathrm{l}$ extraction buffer $(40 \%$ methanol, $40 \%$ acetonitrile, and $0.1 \mathrm{~N}$ formic acid) and incubated on ice for $15 \mathrm{~min}$. Each sample was subjected to three cycles of freezing/thawing using liquid nitrogen and heat block adjusted to $90^{\circ} \mathrm{C}$. After an additional 15-min incubation on ice, the sample was centrifuged at $16,100 \times g$ for $10 \mathrm{~min}$, and $400 \mu \mathrm{l}$ of the supernatant was dried under vacuum. The lyophilized nucleotides were resuspended in $80 \mu \mathrm{l}$ of distilled water.

Quantification of the c-di-GMP was carried out using HPLC coupled with triple quadrupole mass spectrometry. The temperatures of the column oven and autosampler were maintained at 25 and $20^{\circ} \mathrm{C}$, respectively. Ten microliters of the resuspended nucleotides were injected to a Hypersil GOLD HILIC column $(2.1 \times$ $100 \mathrm{~mm}$, particle size $1.9 \mu \mathrm{M}$, pore size $175 \AA$, Thermo Scientific) using Accela 1250 UPLC $^{\mathrm{TM}}$ system (Thermo Fisher Scientific, USA). Separation of c-di-GMP was performed using the following gradient program: solvent A, $20 \mathrm{mM}$ ammonium acetate adjusted to $\mathrm{pH} 8.0$ with ammonium hydroxide; solvent $\mathrm{B}$, acetonitrile $(\mathrm{ACN})$; flow rate $250 \mu \mathrm{l} \mathrm{min}-1$; gradient condition, $0-2 \mathrm{~min}(15 \% \mathrm{~B}), 2-29 \mathrm{~min}$ (15-98\% B), 29-33 min (98\% B), 33-35 min (98-15\% B), 35-45 min (15\% B). Xanthosine $3^{\prime}, 5^{\prime}$-cyclic monophosphate (cXMP; BioLog) was used as anternal standard. The retention times for c-di-GMP and cXMP were 21.5 and $16.8 \mathrm{~min}$, respectively. The analyte detection was performed using TSQ Quantum Access Max (Thermo Scientific). Tune parameters for TSQ were as follows: capillary temperature, $300^{\circ} \mathrm{C}$; vaporization temperature, $250{ }^{\circ} \mathrm{C}$; sheath gas pressure, $30 \mathrm{psi}$; aux gas pressure, $10 \mathrm{psi}$; positive polarity spray voltage, $4.0 \mathrm{kV}$. The samples were monitored with SRM scan mode. The SRM settings for c-di-GMP and cXMP were 
optimized and determined as follows: c-di-GMP: $[\mathrm{M}+\mathrm{H}]^{+} \mathrm{m} / z 691 \rightarrow 152$; collision energy, $38 \mathrm{eV}$; cXMP: $[\mathrm{M}+\mathrm{H}]^{+} \mathrm{m} / z 347 \rightarrow 153$, collision energy, $18 \mathrm{eV}$. The measured intracellular concentration of c-di-GMP was normalized with the protein level.

MST analysis. The binding affinities of PdeS with EIIAGlc and c-di-GMP were measured using a NanoTemper Monolith NT.115 $5^{\text {pico }}$ instrument ${ }^{60}$. Purified GSTPdeS was labeled with NT-647 using a Monolith protein-labeling kit and used at a concentration of $4.875 \mathrm{nM}$. Each unlabeled EIIA ${ }^{\text {Glc }}$ and c-di-GMP was titrated in $1: 1$ serial dilutions in MST-binding buffer (25 mM HEPES-NaOH ( $\mathrm{pH} 7.6), 100$ $\mathrm{mM} \mathrm{NaCl}, 5 \mathrm{mM} \beta$-mercaptoethanol, $0.5 \mathrm{mg} \mathrm{ml}^{-1}$ BSA, 0.05 (v/v) \% Tween 20), with the highest concentration of EIIA Glc at $21.9 \mu \mathrm{M}$ and c-di-GMP at $500 \mu \mathrm{M}$. To prevent degradation of c-di-GMP by PdeS during the assay, their interaction was examined in the buffer without divalent cations which are indispensable for the PDE activity of PdeS. The measurements were performed at $15 \%$ LED power and $30 \%$ MST power at $22^{\circ} \mathrm{C}$

Measurement of biofilm formation. Overnight-grown cells were inoculated in LB medium buffered with $40 \mathrm{mM}$ potassium phosphate ( $\mathrm{pH} \mathrm{7.0)}$ in the absence or presence of sugar and incubated under static conditions in a borosilicate tube at 37 ${ }^{\circ} \mathrm{C}$ for $23 \mathrm{~h}$. After planktonic cells were washed away with PBS, the remaining biofilm-associated cells were stained with $0.1 \%$ crystal violet (CV) for $20 \mathrm{~min}$. After rinses with PBS three times, the CV-stained biofilm was solubilized with $95 \%$ ethanol and measured at $550 \mathrm{~nm}^{61,62}$. Mature biofilm was also visualized as described previously ${ }^{63}$ using confocal laser scanning microscopy (LSM700, Zeiss). $V$. cholerae strains constitutively expressing green fluorescent protein (GFP) were used for biofilm imaging.

Determination of the phosphorylation state of EIIA ${ }^{\text {Glc }}$. The phosphorylation state of EIIA Glc was determined as described previously ${ }^{12,33}$ with some modifications. A $0.2-\mathrm{ml}$ aliquot of cell culture was quenched at $\mathrm{OD}_{600} \sim 0.5$ by adding $20 \mu \mathrm{l}$ of $5 \mathrm{M} \mathrm{NaOH}$ followed by vortexing for $15 \mathrm{~s}$, and then $150 \mu \mathrm{l}$ of $3 \mathrm{M}$ sodium acetate (pH 5.3) and $0.9 \mathrm{ml}$ of ethanol were sequentially added. After incubated at $-80^{\circ} \mathrm{C}$ for $30 \mathrm{~min}$, each sample was centrifuged at $10,000 \times g$ at $4{ }^{\circ} \mathrm{C}$ for $30 \mathrm{~min}$, and the pellet was resuspended in $40 \mu \mathrm{l}$ of SDS sample buffer. A $20-\mu \mathrm{l}$ aliquot of each sample was then resolved by SDS-PAGE using a $4-20 \%$ gradient gel, and EIIA Glc was visualized by western blot using anti-EIIA Glc serum $^{11}$.

Measurement of the cellular protein level by western blot. A V. cholerae strain in which the chromosomal $v c 1710$ was tagged with $3 \times$ FLAG at its C-terminus was grown in $\mathrm{LB}$ medium and aliquots were harvested when $\mathrm{OD}_{600}$ reached $0.4,1.0$, and 1.5 , respectively. After the pellets were resuspended in SDS-loading buffer, cells were lysed by boiling for $5 \mathrm{~min}$. Cell lysates were electrophoresed on a SDS-PAGE gel with various amounts of purified VC1710::3xFLAG protein or EIIA Glc as control. For immunodetection, monoclonal mouse anti-FLAG antibody (SigmaAldrich) or anti-EIIA Glc mouse serum was used. Uncropped blot images were provided as a source data file.

Quantification of $\boldsymbol{V}$. cholerae colonization in the fly intestine. To establish whether sugars could affect the gut colonization of $V$. cholerae strains, fasted adult flies (Drosophila melanogaster w1118) were orally administered a 5\% sugar solution containing $\sim 10^{6}$ cells $_{\mu l^{-1}}$ of the $V$. cholerae strains in the presence of either glucose or mannitol for $24 \mathrm{~h}$, and fed the same, but sterile, medium for another $9 \mathrm{~h}$. After each fly was immersed in $70 \%$ ethanol for $3 \mathrm{~min}$, the surface-sterilized fly was homogenized in $1 \mathrm{ml}$ of sterile PBS and spread on LB agar plates containing $10 \mu \mathrm{g}$ $\mathrm{ml}^{-1}$ of streptomycin to determine CFUs of $V$. cholerae. To image bacterial infection, the whole alimentary canal was dissected at the same time point, and visualized by confocal laser scanning microscopy.

For measurement of food consumption, the feeding assay was performed with a standard method using FD\&C Blue \#1 dye ${ }^{64}$. Flies were orally administered a $5 \%$ sugar (glucose or mannitol) solution containing V. cholerae cells and $0.5 \%(\mathrm{w} / \mathrm{v})$ FD\&C \#1 dye for $2 \mathrm{~h}$. Guts dissected from five flies were homogenized in $200 \mu \mathrm{l}$ of PBS and the homogenate were centrifuged at $8000 \times g$ for $10 \mathrm{~min}$. Food consumption was quantified by measuring absorbance of the supernatant at 625 nm.

Reporting summary. Further information on research design is available in the Nature Research Reporting Summary linked to this article.

\section{Data availability}

The source data underlying Figs. 1a-d, 2b-d, 3a, c, 4a, b, 5a, b, d, and Supplementary Figs. 1a, c, 2, 3, 4a, b, 5a-c, 6, 7a, b and 8 , are provided as a Source Data file.

Received: 29 May 2019; Accepted: 31 October 2019;

Published online: 25 November 2019

\section{References}

1. Flemming, H. C. \& Wingender, J. The biofilm matrix. Nat. Rev. Microbiol. 8 623-633 (2010)

2. O’Toole, G., Kaplan, H. B. \& Kolter, R. Biofilm formation as microbial development. Annu. Rev. Microbiol. 54, 49-79 (2000).

3. Zhu, J. et al. Quorum-sensing regulators control virulence gene expression in Vibrio cholerae. Proc. Natl Acad. Sci. USA 99, 3129-3134 (2002).

4. Zhu, J. \& Mekalanos, J. J. Quorum sensing-dependent biofilms enhance colonization in Vibrio cholerae. Dev. Cell 5, 647-656 (2003).

5. Chua, S. L. et al. Dispersed cells represent a distinct stage in the transition from bacterial biofilm to planktonic lifestyles. Nat. Commun. 5, 4462 (2014).

6. Kaplan, J. B. Biofilm dispersal: mechanisms, clinical implications, and potential therapeutic uses. J. Dent. Res. 89, 205-218 (2010).

7. Karatan, E. \& Watnick, P. Signals, regulatory networks, and materials that build and break bacterial biofilms. Microbiol. Mol. Biol. Rev. 73, 310-347 (2009).

8. Hayes, C. A., Dalia, T. N. \& Dalia, A. B. Systematic genetic dissection of PTS in Vibrio cholerae uncovers a novel glucose transporter and a limited role for PTS during infection of a mammalian host. Mol. Microbiol. 104, 568-579 (2017)

9. Houot, L., Chang, S., Absalon, C. \& Watnick, P. I. Vibrio cholerae phosphoenolpyruvate phosphotransferase system control of carbohydrate transport, biofilm formation, and colonization of the germfree mouse intestine. Infect. Immun. 78, 1482-1494 (2010).

10. Deutscher, J., Francke, C. \& Postma, P. W. How phosphotransferase systemrelated protein phosphorylation regulates carbohydrate metabolism in bacteria. Microbiol. Mol. Biol. Rev. 70, 939-1031 (2006).

11. Nam, T. W., Park, Y. H., Jeong, H. J., Ryu, S. \& Seok, Y. J. Glucose repression of the Escherichia coli sdhCDAB operon, revisited: regulation by the CRP.cAMP complex. Nucleic Acids Res. 33, 6712-6722 (2005).

12. Hogema, B. M. et al. Inducer exclusion in Escherichia coli by non-PTS substrates: the role of the PEP to pyruvate ratio in determining the phosphorylation state of enzyme IIA Glc. Mol. Microbiol. 30, 487-498 (1998).

13. Deutscher, J. et al. The bacterial phosphoenolpyruvate:carbohydrate phosphotransferase system: regulation by protein phosphorylation and phosphorylation-dependent protein-protein interactions. Microbiol. Mol. Biol. Rev. 78, 231-256 (2014)

14. Pickering, B. S., Smith, D. R. \& Watnick, P. I. Glucose-specific enzyme IIA has unique binding partners in the Vibrio cholerae biofilm. MBio 3, e00228-00212 (2012).

15. Pickering, B. S., Lopilato, J. E., Smith, D. R. \& Watnick, P. I. The transcription factor Mlc promotes Vibrio cholerae biofilm formation through repression of phosphotransferase system components. J. Bacteriol. 196, 2423-2430 (2014).

16. Koo, B. M. et al. A novel fermentation/respiration switch protein regulated by enzyme IIA Glc in Escherichia coli. J. Biol. Chem. 279, 31613-31621 (2004).

17. Park, Y. H., Lee, B. R., Seok, Y. J. \& Peterkofsky, A. In vitro reconstitution of catabolite repression in Escherichia coli. J. Biol. Chem. 281, 6448-6454 (2006)

18. Liang, W., Pascual-Montano, A., Silva, A. J. \& Benitez, J. A. The cyclic AMP receptor protein modulates quorum sensing, motility and multiple genes that affect intestinal colonization in Vibrio cholerae. Microbiology 153, 2964-2975 (2007).

19. Silva, A. J. \& Benitez, J. A. Vibrio cholerae biofilms and cholera pathogenesis. PLoS Negl. Trop. Dis. 10, e0004330 (2016).

20. Fong, J. C. \& Yildiz, F. H. Interplay between cyclic AMP-cyclic AMP receptor protein and cyclic di-GMP signaling in Vibrio cholerae biofilm formation. J. Bacteriol. 190, 6646-6659 (2008).

21. Houot, L., Chang, S., Pickering, B. S., Absalon, C. \& Watnick, P. I. The phosphoenolpyruvate phosphotransferase system regulates Vibrio cholerae biofilm formation through multiple independent pathways. J. Bacteriol. 192, 3055-3067 (2010).

22. Park, S., Park, Y. H., Lee, C. R., Kim, Y. R. \& Seok, Y. J. Glucose induces delocalization of a flagellar biosynthesis protein from the flagellated pole. Mol. Microbiol. 101, 795-808 (2016).

23. Park, S. et al. Polar landmark protein HubP recruits flagella assembly protein FapA under glucose limitation in Vibrio vulnificus. Mol. Microbiol. 112, 266-279 (2019).

24. Leng, Y. et al. Regulation of CsrB/C sRNA decay by EIIA Glc of the phosphoenolpyruvate: carbohydrate phosphotransferase system. Mol Microbiol. 99, 627-639 (2016).

25. Jones, M. K., Warner, E. B. \& Oliver, J. D. csrA inhibits the formation of biofilms by Vibrio vulnificus. Appl. Environ. Microbiol. 74, 7064-7066 (2008).

26. Vakulskas, C. A., Potts, A. H., Babitzke, P., Ahmer, B. M. \& Romeo, T. Regulation of bacterial virulence by Csr (Rsm) systems. Microbiol. Mol. Biol. Rev. 79, 193-224 (2015)

27. Beyhan, S., Bilecen, K., Salama, S. R., Casper-Lindley, C. \& Yildiz, F. H. Regulation of rugosity and biofilm formation in Vibrio cholerae: comparison of VpsT and VpsR regulons and epistasis analysis of $v p s T, v p s R$, and hapR. J. Bacteriol. 189, 388-402 (2007). 
28. Houot, L. \& Watnick, P. I. A novel role for enzyme I of the Vibrio cholerae phosphoenolpyruvate phosphotransferase system in regulation of growth in a biofilm. J. Bacteriol. 190, 311-320 (2008).

29. Kierek, K. \& Watnick, P. I. Environmental determinants of Vibrio cholerae biofilm development. Appl. Environ. Microbiol. 69, 5079-5088 (2003).

30. Singh, P. K. et al. Vibrio cholerae combines individual and collective sensing to trigger biofilm dispersal. Curr. Biol. 27, 3359-3366 e3357 (2017).

31. Kim, Y. J. et al. A mammalian insulysin homolog is regulated by enzyme IIA $^{\text {Glc }}$ of the glucose transport system in Vibrio vulnificus. FEBS Lett. 584, 4537-4544 (2010).

32. Galperin, M. Y., Nikolskaya, A. N. \& Koonin, E. V. Novel domains of the prokaryotic two-component signal transduction systems. FEMS Microbiol. Lett. 203, 11-21 (2001).

33. Lee, C. R., Park, Y. H., Min, H., Kim, Y. R. \& Seok, Y. J. Determination of protein phosphorylation by polyacrylamide gel electrophoresis. J. Microbiol. 57, 93-100 (2019).

34. Tchigvintsev, A. et al. Structural insight into the mechanism of c-di-GMP hydrolysis by EAL domain phosphodiesterases. J. Mol. Biol. 402, 524-538 (2010).

35. Roelofs, K. G. et al. Systematic identification of cyclic-di-GMP binding proteins in Vibrio cholerae reveals a novel class of cyclic-di-GMP-binding ATPases associated with Type II secretion systems. PLoS Pathog. 11, e1005232 (2015).

36. Hengge, R. Principles of c-di-GMP signalling in bacteria. Nat. Rev. Microbiol. 7, 263-273 (2009).

37. Cotter, P. A. \& Stibitz, S. c-di-GMP-mediated regulation of virulence and biofilm formation. Curr. Opin. Microbiol. 10, 17-23 (2007).

38. Tischler, A. D. \& Camilli, A. Cyclic diguanylate (c-di-GMP) regulates Vibrio cholerae biofilm formation. Mol. Microbiol. 53, 857-869 (2004).

39. Butler, S. M. \& Camilli, A. Going against the grain: chemotaxis and infection in Vibrio cholerae. Nat. Rev. Microbiol. 3, 611-620 (2005).

40. Blow, N. S. et al. Vibrio cholerae infection of Drosophila melanogaster mimics the human disease cholera. PLoS Pathog. 1, e8 (2005).

41. Mistry, R., Kounatidis, I. \& Ligoxygakis, P. Exploring interactions between pathogens and the Drosophila gut. Dev. Comp. Immunol. 64, 3-10 (2016).

42. Purdy, A. E. \& Watnick, P. I. Spatially selective colonization of the arthropod intestine through activation of Vibrio cholerae biofilm formation. Proc. Natl Acad. Sci. USA 108, 19737-19742 (2011).

43. Kamareddine, L. et al. Activation of Vibrio cholerae quorum sensing promotes survival of an arthropod host. Nat. Microbiol. 3, 243-252 (2018).

44. Buchon, N. et al. Morphological and molecular characterization of adult midgut compartmentalization in Drosophila. Cell Rep. 3, 1725-1738 (2013)

45. Postma, P. W., Lengeler, J. W. \& Jacobson, G. R. Phosphoenolpyruvate: carbohydrate phosphotransferase systems of bacteria. Microbiol. Rev. 57, 543-594 (1993).

46. Blokesch, M. Chitin colonization, chitin degradation and chitin-induced natural competence of Vibrio cholerae are subject to catabolite repression. Environ. Microbiol. 14, 1898-1912 (2012).

47. Ryjenkov, D. A., Simm, R., Romling, U. \& Gomelsky, M. The PilZ domain is a receptor for the second messenger c-di-GMP: the PilZ domain protein YcgR controls motility in enterobacteria. J. Biol. Chem. 281, 30310-30314 (2006).

48. Sauer, K. et al. Characterization of nutrient-induced dispersion in Pseudomonas aeruginosa PAO1 biofilm. J. Bacteriol. 186, 7312-7326 (2004).

49. Gjermansen, M., Ragas, P., Sternberg, C., Molin, S. \& Tolker-Nielsen, T. Characterization of starvation-induced dispersion in Pseudomonas putida biofilms. Environ. Microbiol. 7, 894-906 (2005).

50. Schleheck, D. et al. Pseudomonas aeruginosa PAO1 preferentially grows as aggregates in liquid batch cultures and disperses upon starvation. PLOS ONE 4, e5513 (2009)

51. Schmidt, A. et al. The quantitative and condition-dependent Escherichia coli proteome. Nat. Biotechnol. 34, 104-110 (2016).

52. Liang, W., Silva, A. J. \& Benitez, J. A. The cyclic AMP receptor protein modulates colonial morphology in Vibrio cholerae. Appl. Environ. Microbiol. 73, 7482-7487 (2007).

53. Stutzmann, S. \& Blokesch, M. Circulation of a quorum-sensing-impaired variant of Vibrio cholerae strain C6706 masks important phenotypes. mSphere 1, https://doi.org/10.1128/mSphere.00098-16 (2016).

54. Tamayo, R., Schild, S., Pratt, J. T. \& Camilli, A. Role of cyclic Di-GMP during el tor biotype Vibrio cholerae infection: characterization of the in vivo-induced cyclic Di-GMP phosphodiesterase CdpA. Infect. Immun. 76, 1617-1627 (2008).

55. Schild, S. et al. Genes induced late in infection increase fitness of Vibrio cholerae after release into the environment. Cell Host Microbe 2, 264-277 (2007).

56. Kim, H. M., Park, Y. H., Yoon, C. K. \& Seok, Y. J. Histidine phosphocarrier protein regulates pyruvate kinase A activity in response to glucose in Vibrio vulnificus. Mol. Microbiol. 96, 293-305 (2015).
57. Park, Y. H., Lee, C. R., Choe, M. \& Seok, Y. J. HPr antagonizes the anti- $\sigma 70$ activity of Rsd in Escherichia coli. Proc. Natl Acad. Sci. USA 110, 21142-21147 (2013).

58. Lee, J. W., Park, Y. H. \& Seok, Y. J. Rsd balances (p)ppGpp level by stimulating the hydrolase activity of SpoT during carbon source downshift in Escherichia coli. Proc. Natl Acad. Sci. USA 115, E6845-E6854 (2018).

59. Schmidt, A. J., Ryjenkov, D. A. \& Gomelsky, M. The ubiquitous protein domain EAL is a cyclic diguanylate-specific phosphodiesterase: enzymatically active and inactive EAL domains. J. Bacteriol. 187, 4774-4781 (2005).

60. Bang, I. et al. Biophysical and functional characterization of Norrin signaling through Frizzled4. Proc. Natl Acad. Sci. USA 115, 8787-8792 (2018).

61. Lee, K. J., Kim, J. A., Hwang, W., Park, S. J. \& Lee, K. H. Role of capsular polysaccharide (CPS) in biofilm formation and regulation of CPS production by quorum-sensing in Vibrio vulnificus. Mol. Microbiol. 90, 841-857 (2013).

62. Watnick, P. I. \& Kolter, R. Steps in the development of a Vibrio cholerae El Tor biofilm. Mol. Microbiol. 34, 586-595 (1999).

63. Townsley, L. \& Yildiz, F. H. Temperature affects c-di-GMP signalling and biofilm formation in Vibrio cholerae. Environ. Microbiol. 17, 4290-4305 (2015).

64. Ha, E. M. et al. Coordination of multiple dual oxidase-regulatory pathways in responses to commensal and infectious microbes in drosophila gut. Nat. Immunol. 10, 949-957 (2009).

65. Watnick, P. I., Fullner, K. J. \& Kolter, R. A role for the mannose-sensitive hemagglutinin in biofilm formation by Vibrio cholerae El Tor. J. Bacteriol. 181, 3606-3609 (1999).

\section{Acknowledgements}

This work was supported by National Research Foundation Grants NRF2018R1A5A1025077 and NRF-2019R1A2C2004143 funded by the Ministry of Science and ICT. W.-J.L. and K.-A.L. are supported by the national creative research initiative program (2015R1A3A2033475) and basic science research program (NRF2019R1I1A1A01059606) of the National Research Foundation of South Korea. K.H. and H.-I.H. were supported by the BK21 plus program of the National Research Foundation of South Korea.

\section{Author contributions}

K.H., Y.-H.P., H.-I.H., and Y.-J.S. designed the study. K.H., Y.-H.P., K.-A.L., and J.K. performed the experiments. K.H., Y.-H.P., K.-A.L., B.-G.K., W.-J.L., and Y.-J.S. analyzed data. K.H., H.-I.H., W.-J.L. and Y.-J.S. wrote the paper.

\section{Competing interests}

The authors declare no competing interests.

\section{Additional information}

Supplementary information is available for this paper at https://doi.org/10.1038/s41467019-13353-5.

Correspondence and requests for materials should be addressed to Y.-J.S

Peer review information Nature Communications thanks Josef Deutscher and the other, anonymous, reviewers for their contribution to the peer review of this work. Peer reviewer reports are available.

Reprints and permission information is available at http://www.nature.com/reprints

Publisher's note Springer Nature remains neutral with regard to jurisdictional claims in published maps and institutional affiliations.

Open Access This article is licensed under a Creative Commons Attribution 4.0 International License, which permits use, sharing, adaptation, distribution and reproduction in any medium or format, as long as you give appropriate credit to the original author(s) and the source, provide a link to the Creative Commons license, and indicate if changes were made. The images or other third party material in this article are included in the article's Creative Commons license, unless indicated otherwise in a credit line to the material. If material is not included in the article's Creative Commons license and your intended use is not permitted by statutory regulation or exceeds the permitted use, you will need to obtain permission directly from the copyright holder. To view a copy of this license, visit http://creativecommons.org/ licenses/by/4.0/.

(C) The Author(s) 2019 